\title{
STABILIZATION OF NONAFFINE NONLINEAR SYSTEMS USING TIME-SCALE SEPARATION
}

\author{
Naira Hovakimyan* Eugene Lavretsky ** \\ Amol Sasane ${ }^{* * *}$ \\ * Aerospace and Ocean Engineering, Virginia Tech, 215 \\ Randolph Hall, Blacksburg VA 24061, U.S.A. \\ ** Phantom Works, The Boeing Company, U.S.A. \\ ${ }^{* * *}$ Department of Mathematics, London School of \\ Economics, Houghton Street, London WC2A 2AE, U.K.
}

\begin{abstract}
In this article, a new control design is proposed for the stabilization problem for large class of nonaffine nonlinear systems using time-scale separation and a key result from singular perturbation theory. Copyright@IFAC 2005.
\end{abstract}

Keywords: nonlinear control systems, stabilization, singular perturbation method

\section{INTRODUCTION}

Most of the research for control design of nonlinear systems has been done for systems that are affine in the control variables. However, many practical applications (chemical reactions and $\mathrm{PH}$ neutralization, aircraft dynamics and so on) give rise to nonaffine nonlinear systems. Since the input does not appear linearly, this makes direct feedback linearization difficult. Also, even if it is known that the inverse of such a system exists, it is often impossible to construct it analytically. Consequently conventional control designs are not possible for nonaffine nonlinear systems.

In this paper, a new control design is proposed for a large class of nonaffine nonlinear systems. In the following paragraph the heuristics behind this approach are briefly illustrated, by considering a simple scalar example.

Consider the scalar nonlinear system

$$
\dot{x}=f(x, u),
$$

$x(t) \in \mathbb{R}, u(t) \in \mathbb{R}, t \geq 0$. The control objective is to design $u$ to track the state of the asymptotically stable reference system

$$
\dot{x}=a x,
$$

with $a<0$. One would like to solve for $u$ from the equation

$$
f(x, u)=a x .
$$

Now suppose that $\operatorname{sign}\left(\frac{\partial f}{\partial u}\right)$ is constant, and consider the system

$$
\epsilon \dot{u}=-\operatorname{sign}\left(\frac{\partial f}{\partial u}\right)(f(x, u)-a x),
$$

with $\epsilon \ll 1$. This equation represents "fast" dynamics as compared to the "slow" dynamics of $\dot{x}=f(x, u)$. Let $x$ be fixed, and let $u$ satisfy (1). Then intuitively we would expect that if $u$ had a limit as $t \rightarrow \infty$, it would satisfy the static equation

$$
f(x, u(\infty))=a x .
$$

Therefore the solution to the fast dynamical equation (1), with a small enough $\epsilon$, can be viewed to be an approximation of the solution to the equation $f(x, u)=a x$, and so we arrive at the following closed loop system:

$$
\begin{aligned}
\dot{x} & =f(x, u), \\
\epsilon \dot{u} & =-\operatorname{sign}\left(\frac{\partial f}{\partial u}\right)(f(x, u)-a x) .
\end{aligned}
$$


Hence the need of solving the implicit equation

$$
f(x, u)=a x
$$

is eliminated by introducing the fast dynamics given by (1).

As a concrete example, consider the problem of stabilization of the scalar nonlinear system given by

$$
\dot{x}=e^{x}+u+u^{2} \tanh (u) .
$$

A stabilizing dynamic inversion controller can be obtained by solving the following equation for $u$ :

$$
e^{x}+u+u^{2} \tanh (u)=-x .
$$

It can be checked that for $u \in \mathbb{R}, \frac{\partial f}{\partial u}$ has a constant sign. Hence the system is controllable. Notice however that the equation (3) is not invertible explicitly, and hence the precise dynamic inversion solution for $u$ cannot be found. So we approximate the dynamic inversion solution via time-scale separation. Consider the following fast dynamics:

$\epsilon \dot{u}=-\operatorname{sign}\left(\frac{\partial f}{\partial u}\right)\left(e^{x}+u+u^{2} \tanh (u)+x\right), \quad \epsilon \ll 1$.

When $\epsilon=0$, the relationship in (4) reduces to the

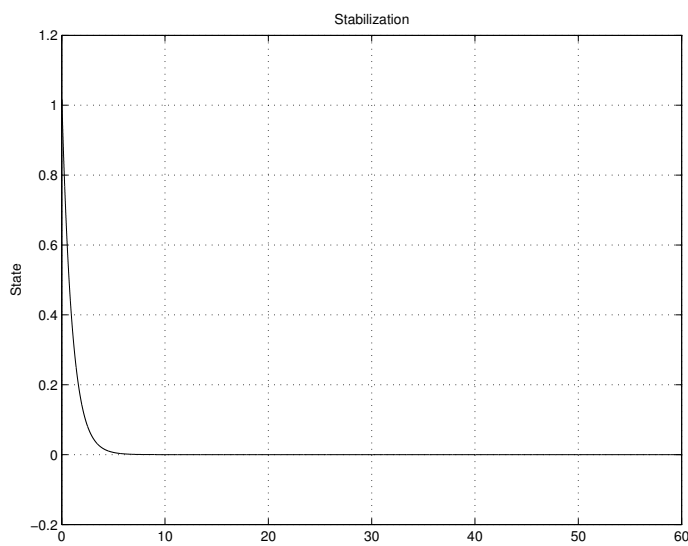

Fig. 1. Stabilization

algebraic relationship in (3), the solution of which renders the system (2) asymptotically stable:

$$
\dot{x}=-a x \text {. }
$$

For a suitably chosen small $\epsilon$, the solution of differential equation (4), achieves asymptotic stabilization of the system (2), as shown in Figure 1.

The outline of this paper is as follows. First in $\S 2$, we recall a few definitions and theorems on nonaffine nonlinear systems and singular perturbations. We give our main result concerning stabilization $\S 3$.

\section{PRELIMINARIES ON SINGULAR PERTURBATIONS}

In this section we recall Tikhonov's theorem (Theorem 2.1 below) on singular perturbations. We will use this key theorem to obtain our main result Theorem 3.1 in the next section. Consider the problem of solving the system

$$
\Sigma_{0}:\left\{\begin{array}{l}
\dot{x}(t)=f(t, x(t), u(t), \epsilon), \quad x(0)=\xi(\epsilon) \\
\epsilon \dot{u}(t)=g(t, x(t), u(t), \epsilon), u(0)=\eta(\epsilon)
\end{array}\right\},
$$

where $\xi: \epsilon \mapsto \xi(\epsilon)$ and $\eta: \epsilon \mapsto \eta(\epsilon)$ are smooth. We assume that $f$ and $g$ are continuously differentiable in their arguments for $(t, x, u, \epsilon) \in$ $\left[0, t_{1}\right] \times D_{x} \times D_{u} \times\left[0, \epsilon_{0}\right]$, where $D_{x} \subset \mathbb{R}^{n}$ and $D_{u} \subset \mathbb{R}^{m}$ are domains. We also assume that $\Sigma_{0}$ is in standard form, that is,

$$
0=g(t, x, u, 0)
$$

has $k \geq 1$ isolated real roots

$$
u=h_{i}(t, x), \quad i \in\{1, \ldots, k\}
$$

for each $(t, x) \in\left[0, t_{1}\right] \times D_{x}$. We choose one particular $i$, which is fixed. We drop the subscript henceforth. The reduced order system is given by

$\Sigma_{00}: \quad \dot{x}(t)=f(t, x(t), h(t, x(t)), 0), \quad x(0)=\xi(0)$,

and the solution is denoted by $x_{\mathrm{r}}$. Let $\eta(0)=\eta_{0}$ and $\xi(0)=\xi_{0}$. The boundary layer system is given by

$$
\Sigma_{b}:\left\{\begin{array}{l}
\frac{d y}{d \tau}=g(t, x, y+h(t, x), 0), \quad \tau=\frac{t}{\epsilon}, \\
y(0)=\eta_{0}-h\left(0, \xi_{0}\right), \quad(t, x) \in\left[0, t_{1}\right] \times D_{x} .
\end{array}\right.
$$

The equilibrium point $y=0$ of $\Sigma_{b}$ is exponentially stable uniformly in $(t, x) \in\left[0, t_{1}\right] \times D_{x}$, if there exist positive constants $M, \omega$ and $\rho_{0}$ such that the solutions satisfy

$$
\|y(\tau)\| \leq M e^{-\omega \tau}\|y(0)\|
$$

for all $y_{0}$ such that $\|y(0)\|<\rho_{0}$, for all $(t, x) \in$ $\left[0, t_{1}\right] \times D_{x}$, and for all $\tau \geq 0$. With this set up, we recall the following Tikhonov's theorem; see for instance Theorem 11.2 on page 439 of (Khalil, 2002):

Theorem 2.1. Consider the singular perturbation system $\Sigma_{0}$ given by equation (5) and let $u=$ $h(t, x)$ be an isolated root of (6). Assume that the following conditions are satisfied for all

$$
[t, x, u-h(t, x), \epsilon] \in[0, \infty) \times D_{x} \times D_{y} \times\left[0, \epsilon_{0}\right]
$$

for some domains $D_{x} \subset \mathbb{R}^{n}$ and $D_{y} \subset \mathbb{R}^{m}$, which contain their respective origins:

A1. On any compact subset of $D_{x} \times D_{y}$, the functions $f, g$, their first partial derivatives with respect to $(x, u, \epsilon)$, and the first partial derivative of $g$ with respect to $t$ are continuous and bounded, $h(t, x)$ and $\left[\frac{\partial g}{\partial u}(t, x, u, 0)\right]$ have bounded 
first derivatives with respect to their arguments, $\left[\frac{\partial f}{\partial x}(t, x, h(t, x))\right]$ is Lipschitz in $x$, uniformly in $t$, and the initial data given by $\xi$ and $\eta$ are smooth functions of $\epsilon$.

A2. The origin is an exponentially stable equilibrium point of the reduced system $\Sigma_{00}$ given by equation (7). There exists a Lyapunov function $V:[0, \infty) \times D_{x} \rightarrow[0, \infty)$ that satisfies

$$
\begin{gathered}
W_{1}(x) \leq V(t, x) \leq W_{2}(x) \\
\frac{\partial V}{\partial t}(t, x)+\frac{\partial V}{\partial x}(t, x) f(t, x, h(t, x), 0) \leq-W_{3}(x)
\end{gathered}
$$

for all $(t, x) \in[0, \infty) \times D_{x}$, where $W_{1}, W_{2}, W_{3}$ are continuous positive definite functions on $D_{x}$, and let $c$ be a nonnegative number such that $\left\{x \in D_{x} \mid W_{1}(x) \leq c\right\}$ is a compact subset of $D_{x}$.

A3. The origin is an equilibrium point of the boundary layer system $\Sigma_{b}$ given by equation (8) which is exponentially stable uniformly in $(t, x)$.

Let $R_{y} \subset D_{y}$ denote the region of attraction of the autonomous system

$$
\frac{d y}{d \tau}=g\left(0, \xi_{0}, y+h\left(0, \xi_{0}\right), 0\right), \quad \tau=\frac{t}{\epsilon},
$$

and let $\Omega_{y}$ be a compact subset of $R_{y}$. Then for each compact set

$$
\Omega_{x} \subset\left\{x \in D_{x} \mid W_{2}(x) \leq \rho c, 0<\rho<1\right\},
$$

there exists a positive constant $\epsilon_{*}$ such that for all $t \geq 0, \xi_{0} \in \Omega_{x}, \eta_{0}-h\left(0, \xi_{0}\right) \in \Omega_{y}$ and $0<\epsilon<\epsilon_{*}$, $\Sigma_{0}$ has a unique solution $x_{\epsilon}$ on $[0, \infty)$ and

$$
x_{\epsilon}(t)-x_{\mathrm{r}}(t)=O(\epsilon)
$$

holds uniformly for $t \in[0, \infty)$, where $x_{\mathbf{r}}(t)$ denotes the solution of the reduced system $\Sigma_{00}$ given by (7).

Remark 2.1 One can use a Lyapunov argument to check Assumption A3: if there is a Lyapunov function $V:[0, \infty) \times D_{x} \times D_{y}$ that satisfies

$$
\begin{gathered}
c_{1}\|y\|^{2} \leq V(t, x, y) \leq c_{2}\|y\|^{2} \\
\frac{\partial V}{\partial y} g(x, y+h(t, x)) \leq-c_{3}\|y\|^{2}
\end{gathered}
$$

for all $(t, x, y) \in D_{x} \times D_{y}$, then Assumption A3 is satisfied.

\section{STABILIZATION}

In this section we give our main result concerning stabilization (Theorem 3.1).

Consider the following nonlinear system in nonaffine normal form:

$$
\begin{aligned}
& \begin{aligned}
{\left[\begin{array}{l}
\dot{x}_{1,1}(t) \\
\vdots \\
\dot{x}_{1, r_{1}-1}(t) \\
\dot{x}_{1, r_{1}}(t)
\end{array}\right]=} & {\left[\begin{array}{llll}
0 & 1 & & \\
\vdots & & \ddots & \\
0 & & & 1 \\
0 & 0 & \ldots & 0
\end{array}\right]\left[\begin{array}{l}
x_{1,1}(t) \\
\vdots \\
x_{1, r_{1}-1}(t) \\
x_{1, r_{1}}(t)
\end{array}\right] } \\
& +\left[\begin{array}{c}
0 \\
\vdots \\
f_{1}(z(t), x(t), u(t))
\end{array}\right]
\end{aligned} \\
& \begin{aligned}
{\left[\begin{array}{l}
\dot{x}_{m, 1}(t) \\
\vdots \\
\dot{x}_{m, r_{m}-1}(t) \\
\dot{x}_{m, r_{m}}(t)
\end{array}\right]=} & {\left[\begin{array}{llll}
0 & 1 & & \\
\vdots & & \ddots & \\
0 & & & 1 \\
0 & 0 & \ldots & 0
\end{array}\right]\left[\begin{array}{l}
x_{m, 1}(t) \\
\vdots \\
x_{m, r_{m}-1}(t) \\
x_{m, r_{m}}(t)
\end{array}\right] } \\
& +\left[\begin{array}{c}
0 \\
\vdots \\
0 \\
f_{m}(z(t), x(t), u(t))
\end{array}\right] \\
\dot{z}(t) & =\zeta(z(t), x(t), u(t))
\end{aligned}
\end{aligned}
$$

where $x=\left[\begin{array}{lllllll}x_{1,1} & \ldots & x_{1, r_{1}} \ldots & x_{m, 1} & \ldots & x_{m, r_{m}}\end{array}\right]^{\top}$ and $u=\left[\begin{array}{lll}u_{1} & \ldots & u_{m}\end{array}\right]^{\top}$.

The control objective is to design $u$ such that the state in equation (10) tracks the state of the following reference model:

$$
\dot{x}_{k, r_{k}}(t)=A_{r, k} x_{k}, \quad k \in\{1, \ldots, m\}
$$

where

$$
A_{r, k}=\left[\begin{array}{cccc}
0 & 1 & & \\
\vdots & & \ddots & \\
0 & & & 1 \\
a_{k, 1} & a_{k, 2} & \ldots & a_{k, r_{k}}
\end{array}\right], k \in\{1, \ldots, m\}
$$

are all Hurwitz. We need to solve for $u$ from the equations:

$$
f_{k}(z, x, u)=a_{k, 1} x_{k, 1}+\cdots+a_{k, r_{k}} x_{k, r_{k}},
$$

$k \in\{1, \ldots, m\}$. So we introduce the appropriate fast dynamics, and applying Theorem 2.1, we obtain the following theorem:

Theorem 3.1. Consider the system given by (10) along with the following fast dynamics:

$$
\epsilon \dot{u}(t)=P \mathbf{f}(z(t), x(t), u(t))
$$

where

$$
\begin{aligned}
& \mathbf{f}(z, x, u)= \\
& {\left[\begin{array}{c}
f_{1}(z, x, u)-a_{1,1} x_{1,1}-\cdots-a_{1, r_{1}} x_{1, r_{1}} \\
\vdots \\
f_{m}(z, x, u)-a_{m, 1} x_{m, 1}-\cdots-a_{m, r_{m}} x_{m, r_{m}}
\end{array}\right],} \\
& x=\left[\begin{array}{lll}
x_{1,1} \ldots x_{1, r_{1}} \ldots x_{m, 1} \ldots x_{m, r_{m}}
\end{array}\right]^{\top}, \\
& u=\left[\begin{array}{lll}
u_{1} \ldots u_{m} & \top
\end{array}\right]^{\top}, \\
& P \in \mathbb{R}^{m \times m}, \text { with } x(0)=x_{0} \in \mathbb{R}^{r_{1}+\cdots+r_{m}}, z(0)= \\
& z_{0} \in \mathbb{R}^{n-\left(r_{1}+\cdots+r_{m}\right)}, u(0)=u_{0} \in \mathbb{R}^{m} \text { and }
\end{aligned}
$$




$$
A_{\mathrm{r}, k}=\left[\begin{array}{cccc}
0 & 1 & & \\
\vdots & & \ddots & \\
0 & & & 1 \\
a_{k, 1} & a_{k, 2} & \ldots & a_{k, r_{k}}
\end{array}\right], k \in\{1, \ldots, m\}
$$

are Hurwitz matrices. Let $u=h(z, x)$ be an isolated root of the equation

$$
\mathbf{f}(z, x, u)=0 .
$$

Assume that the following conditions are satisfied for all

$[t,(z, x), u-h(z, x), \epsilon] \in[0, \infty) \times D_{z, x} \times D_{y} \times\left[0, \epsilon_{0}\right]$ for some domains $D_{z, x} \subset \mathbb{R}^{n}$ and $D_{y} \subset \mathbb{R}^{m}$, which contain their respective origins:

B1. On any compact subset of $D_{z, x} \times D_{y}$, the functions $f_{k}, k \in\{1, \ldots, m\}$, and their first partial derivatives with respect to $(z, x, u)$, are continuous and bounded, $h(z, x)$ has bounded first derivatives with respect to $x$ and $z$, and the maps

$$
(z, x) \mapsto\left[\frac{\partial f_{k}}{\partial z}(z, x, h(z, x)) \frac{\partial f_{k}}{\partial x}(z, x, h(z, x))\right],
$$

$k \in\{1, \ldots, m\}$ are Lipschitz.

B2. The origin is an exponentially stable equilibrium point of $\dot{z}=\zeta(z, 0, h(z, 0))$. The map $(z, x) \mapsto \zeta(z, x, h(z, x))$ is continuously differentiable and Lipschitz in $(z, x) \in D_{z, x}$.

B3. There exists a positive number $c$ such that

$$
\begin{aligned}
& \frac{\partial \mathbf{f}}{\partial u}(z, x, y+h(z, x))^{\top} P^{\top}+P \frac{\partial \mathbf{f}}{\partial u}(z, x, y+h(z, x)) \\
& \quad<-c I<0
\end{aligned}
$$

for all $(z, x) \in D_{z, x}, y \in D_{y}$.

Let $R_{y} \subset D_{y}$ denote the region of attraction of the autonomous system

$$
\frac{d y}{d \tau}=P \mathbf{f}\left(z_{0}, x_{0}, y+h\left(z_{0}, x_{0}\right)\right), \quad \tau=\frac{t}{\epsilon}
$$

and let $\Omega_{y}$ be a compact subset of $R_{y}$. Then for each compact set $\Omega_{z, x} \subset D_{z, x}$ there exists a positive constant $\epsilon_{*}$ and a $T \geq 0$ such that for all $\left(z_{0}, x_{0}\right) \in \Omega_{z, x}, u_{0}-h\left(z_{0}, x_{0}\right) \in \Omega_{y}$ and $0<\epsilon<\epsilon_{*}$, the system of equations given by (10)-(11) has a unique solution $x_{\epsilon}, z_{\epsilon}$ on $[0, \infty)$ and

$$
x_{\epsilon}(t)=x_{\mathrm{r}}(t)+O(\epsilon)
$$

holds uniformly for $t \in[T, \infty)$, where $x_{\mathrm{r}}$ denotes the solution to

$$
\dot{x}_{\mathrm{r}}(t)=A_{\mathrm{r}} x_{\mathrm{r}}(t), \quad x_{\mathrm{r}}(0)=x_{\mathrm{r}, 0},
$$

and $A_{\mathrm{r}}$ is the block diagonal matrix given by

$$
A_{\mathrm{r}}=\left[\begin{array}{lll}
A_{\mathrm{r}, 1} & & \\
& \ddots & \\
& & A_{\mathrm{r}, m}
\end{array}\right] \text {. }
$$

Furthermore,

$$
z_{\epsilon}(t)=z_{\mathrm{r}}(t)+O(\epsilon)
$$

holds uniformly for $t \in[0, \infty)$, where $z_{\mathrm{r}}$ denotes the solution of

$\dot{z}_{\mathrm{r}}(t)=\zeta\left(z_{\mathrm{r}}(t), e^{t A_{\mathrm{r}}} x_{0}, h\left(z_{\mathbf{r}}(t), e^{t A_{\mathrm{r}}} x_{0}\right)\right), z_{\mathrm{r}}(0)=z_{0}$.

Proof First we verify that Assumptions A1, A2, A3 in Theorem 2.1 are satisfied. Since $f=$ $\left[\begin{array}{lll}f_{1} & \ldots & f_{m}\end{array}\right]^{\top}$ and $h$ are independent of $t$, Assumption B1 clearly implies that A1 holds.

We now show that Assumption A2 holds. From Lemma 4.6 on page 176 of (Khalil, 2002), it follows that the system

$$
\dot{z}(t)=\zeta(z(t), x(t), h(z(t), x(t)))
$$

(with $x$ viewed as the input) is input to state stable. Thus there exists class $\mathcal{K}$ and class $\mathcal{K} \mathcal{L}$ functions $\gamma$ and $\beta$, respectively, such that

$$
\|z(t)\| \leq \beta\left(\left\|z\left(t_{0}\right)\right\|, t-t_{0}\right)+\gamma\left(\sup _{t_{0} \leq \tau \leq t}\|x(\tau)\|\right)
$$

for all $t \geq t_{0}, t_{0} \in[0, \infty)$. Furthermore from the proof of Lemma 4.6 of (Khalil, 2002), it follows that $\gamma(r)=c r$, for some constant $c>0$. Using the fact that the unforced system $\dot{z}=\zeta(z, 0, h(z, 0))$ has 0 as an exponentially stable equilibrium point, it can be seen from the proof of Lemma 4.6 of (Khalil, 2002) that $\beta(r, t)=k r e^{-\omega t}$ for some positive constants $k$ and $\omega$. Thus the solution to the system of equations

$$
\begin{aligned}
& \dot{x}(t)=A_{\mathrm{r}} x(t), \quad x(0)=x_{0} \\
& \dot{z}(t)=\zeta(z(t), x(t), h(z(t), x(t))), z(0)=z_{0}
\end{aligned}
$$

satisfies $\|x(t)\| \leq\left\|x_{0}\right\| c_{1} e^{-\omega_{0} t}$ and $\|z(t)\| \leq$ $\left(\left\|x_{0}\right\|+\left\|z_{0}\right\|\right) c_{2} e^{-\omega_{0} t}$ for all $t \geq 0$ and for some $\omega_{0}>0$. Hence the origin $(0,0)$ is an exponentially stable equilibrium point of (15)-(16). From a converse Lyapunov theorem (Theorem 4.14 on pages 162-163 of (Khalil, 2002)), it follows that there exists a Lyapunov function $V:[0, \infty) \times D_{z, x} \rightarrow \mathbb{R}$ such that

$$
\begin{gathered}
w_{1}\|(z, x)\|^{2} \leq V(t,(z, x)) \leq w_{2}\|(z, x)\|^{2} \\
\frac{\partial V}{\partial t}(t,(z, x))+\nabla_{z, x} V \cdot F(z, x) \leq-w_{3}\|(z, x)\|^{2},
\end{gathered}
$$

where

$$
F(z, x)=\left[\begin{array}{c}
A_{\mathrm{r}} x \\
\zeta(z, x, h(z, x))
\end{array}\right] .
$$

We note that any positive $c$ can be chosen in A2 of Theorem 2.1, and so a compact $\Omega_{z, x} \subset\{(z, x) \in$ $\left.D_{z, x} \mid W_{2}(z, x) \leq \rho c, 0<\rho<1\right\}$ can be chosen to be any subset of $D_{z, x}$.

In light of the Remark 2.1 (following Theorem 2.1 in $\S 2)$, it is easy to see that Assumption B3 implies A3. Indeed, consider the candidate Lyapunov function

$V(z, x, y)=\langle\mathbf{f}(z, x, y+h(z, x)), \mathbf{f}(z, x, y+h(z, x))\rangle$ 
for the dynamics

$$
\frac{d y}{d \tau}=P \mathbf{f}(z, x, y+h(z, x)), \quad \tau=\frac{t}{\epsilon} .
$$

From Assumption B1 it follows that $\mathbf{f}$ is locally Lipschitz in $y$ uniformly in $(z, x)$. Furthermore, since $\mathbf{f}(z, x, h(z, x))=0$, it follows that given any compact subsets $\Omega_{z, x} \subset D_{z, x}$ and $\Omega_{y} \subset D_{y}$, there exist constants $c_{1}$ and $c_{2}$ such that

$$
c_{1}\|y\|^{2} \leq V(z, x, y) \leq c_{2}\|y\|^{2},
$$

for all $(z, x, y) \in \Omega_{z, x} \times \Omega_{y}$. Moreover,

$$
\begin{aligned}
\dot{V} & =\left\langle\left(\left[\frac{\partial \mathbf{f}}{\partial u}\right]^{\top} P^{\top}+P\left[\frac{\partial \mathbf{f}}{\partial u}\right]\right) \mathbf{f}, \mathbf{f}\right\rangle \\
& <-c\langle\mathbf{f}(z, x, y+h(z, x)), \mathbf{f}(z, x, y+h(z, x))\rangle \\
& <-c c_{2}\|y\|^{2} .
\end{aligned}
$$

Hence Theorem 2.1 applies and so it follows that for each compact set $\Omega_{z, x} \subset D_{z, x}$ there exists a positive constant $\epsilon_{*}$ and such that for all $\left(z_{0}, x_{0}\right) \in$ $\Omega_{z, x}, u_{0}-h\left(z_{0}, x_{0}\right) \in \Omega_{y}$ and $0<\epsilon<\epsilon_{*}$, the system of equations given by (10)-(11) has a unique solution $x_{\epsilon}, z_{\epsilon}$ on $[0, \infty)$ and

$$
\begin{aligned}
& x_{\epsilon}(t)=e^{t A_{\mathrm{r}}} x_{\mathrm{r}, 0}+O(\epsilon), \\
& z_{\epsilon}(t)=z_{\mathrm{r}}(t)+O(\epsilon)
\end{aligned}
$$

hold uniformly for $t \in[0, \infty)$, where $z_{\mathrm{r}}$ denotes the solution of

$$
\begin{aligned}
& \dot{x}(t)=A_{\mathrm{r}} x(t), \quad x(0)=x_{0}, \\
& \dot{z}(t)=\zeta(z(t), x(t), h(z(t), x(t))), \quad z(0)=z_{0},
\end{aligned}
$$

Hence (14) holds uniformly for $t \in[0, \infty)$. Choose $T \geq 0$ such that

$$
\left\|e^{t A_{\mathrm{r}}} x_{0}-e^{t A_{\mathrm{r}}} x_{\mathrm{r}, 0}\right\| \leq \epsilon,
$$

and this proves that (13) holds uniformly for $t \in[T, \infty)$.

Remark 3.1 1. The verification of Assumption $\mathrm{B} 3$ is easy in many cases. We list a few instances below.

(a) Let co $\operatorname{spec}(M)$ denote the convex hull of the spectrum of the square matrix $M$. If the map

$(z, x, y) \mapsto \operatorname{dist}\left(\operatorname{cospec}\left(\left[\frac{\partial \mathbf{f}}{\partial u}(z, x, y+h(z, x))\right]\right), i \mathbb{R}\right)$

is bounded below by a positive number, then we can take $P=-\operatorname{sign}\left(\operatorname{tr}\left[\frac{\partial \mathbf{f}}{\partial u}\right]\right)$, where $\operatorname{tr}(\cdot)$ denotes the trace function.

(b) In the case when there exist $d_{1}, \ldots, d_{m}>$ 0 and $\delta_{1}, \ldots, \delta_{m} \in[0,1)$ such that for $k \in$ $\{1, \ldots, m\}$

$$
\left|\left[\frac{\partial \mathbf{f}}{\partial u}\right]_{k, k}\right| \geq d_{k} \text { and } \sum_{\substack{l=1 \\ l \neq k}}^{m}\left|\left[\frac{\partial \mathbf{f}}{\partial u}\right]_{k, l}\right| \leq \delta_{k} d_{k}
$$

then using Gershgorin's circle theorem; see for instance (Brualdi and Mellendorf 1994), it can be seen that $P$ can be chosen to be the diagonal matrix

$$
\left[\begin{array}{ccc}
-\operatorname{sign}\left(\left[\frac{\partial \mathbf{f}}{\partial u}\right]_{1,1}\right) & \\
& \ddots & \\
& & -\operatorname{sign}\left(\left[\frac{\partial \mathbf{f}}{\partial u}\right]_{m, m}\right)
\end{array}\right] .
$$

A special case of the above diagonal dominance is when $f_{1}, \ldots, f_{m}$ depend only on $u_{1}, \ldots, u_{m}$, respectively, that is, when $\frac{\partial f_{i}}{\partial u_{j}}=0$ for all $i$ and $j$ such that $i \neq j$, and so $\left[\frac{\partial \mathbf{f}}{\partial u}\right]$ is diagonal. If

$$
(z, x, y) \mapsto \operatorname{sign}\left(\frac{\partial f_{k}}{\partial u_{k}}(z, x, y+h(z, x))\right)
$$

is bounded away from zero, then we can take $P$ to be equal to the diagonal matrix given in (17).

2. We note that the result of Theorem 3.1 is semiglobal: indeed, if $D_{z, x}=\mathbb{R}^{n}$, then for every compact subset $\Omega_{z, x}$ of $\mathbb{R}^{n}$, there exists an $\epsilon_{*}$ such that with the feedback given by (11) we get the tracking performance specified in (15)-(16).

\section{CONCLUSION}

In this article, a new control design has been given for solving the stabilization problem for a class of nonaffine nonlinear systems using time-scale separation and a key result from singular perturbation theory. An illustrative example was given, and some conditions were given under which the assumptions in the main theorem can be verified easily.

\section{REFERENCES}

Brualdi, R.A. and Mellendorf, S. (1994). Regions in the complex plane containing the eigenvalues of a matrix. The American Mathematical Monthly, 101, 975-985.

Khalil, H.K. (2002). Nonlinear Systems. Prentice Hall, London.

Nijmeijer, H. and van der Schaft, A.J. (1990). Nonlinear Dynamical Control Systems. Springer, Berlin.

Slotine, J.E. and Li, W. (1991). Applied Nonlinear Control. Prentice Hall, London. 